\title{
Oxidative stability of the lipid fraction in cookies - the EPR study
}

Katarzyna Zawada, Mariola Kozłowska, Anna Żbikowska

\begin{abstract}
Cookies are a group of convenient food products that are popular among consumers. They may contain high amounts of fats, which can be prone to oxidation. To retard the oxidative deterioration, synthetic and natural antioxidants may be added. Herb and spice extracts can be sources of natural biologically active substances with antioxidant activity. In this work, electron paramagnetic resonance spectroscopy was used to monitor the lipid oxidation in cookies with rosemary and thyme extracts subjected to the storage in elevated temperature. It was shown that thyme extract can be used as a natural antioxidant source for the preparation of bakery products, while the rosemary extract should be used with care in fat-rich products exposed to high temperatures.
\end{abstract}

Key words: cookies $\bullet$ EPR spectroscopy $\bullet$ oxidative stability $\bullet$ rosemary $\bullet$ thyme

\section{K. Zawada}

Department of Physical Chemistry,

Faculty of Pharmacy,

Medical University of Warsaw,

1 Banacha Str., 02-097 Warsaw, Poland,

Tel./Fax: +48 22572 0950,

E-mail: katarzyna.zawada@wum.edu.pl

M. Kozłowska

Department of Chemistry,

Faculty of Food Sciences,

Warsaw University of Life Sciences (WULS-SGGW), 159C Nowoursynowska Str., 02-776 Warsaw, Poland

\section{A. Żbikowska}

Department of Food Technology,

Faculty of Food Sciences,

Warsaw University of Life Sciences (WULS-SGGW), 159C Nowoursynowska Str., 02-776 Warsaw, Poland

Received: 24 September 2014

Accepted: 30 January 2015

\section{Introduction}

Bakery products such as cookies belong to the group of convenient food products that are often sought by consumers. Cookies may contain high amounts of fats, which should have high oxidative stability and suitable plasticity. The type of fat has a significant impact not only on physical and sensory properties of high fat products, but also on their nutritional characteristic. Particularly important is the composition of fatty acids, including the content of polyunsaturated fatty acids that are important nutrients, but on the other hand, are prone to oxidative reactions. These reactions decrease the quality and functional properties of cookies, and reduce their shelf-life [1]. To retard the oxidative deterioration, synthetic and natural antioxidants may be added. The use of synthetic antioxidants such as butylated hydroxyanisole (BHA) or butylated hydroxytoluene (BHT) is restricted because of their possible toxicity in high doses [2, 3]. Therefore herbs and spices, as well as their extracts, may be important ingredients of bakery products, acting as sources of biologically active substances with antioxidant activity. The most important group of these substances are polyphenols. In addition to antioxidant activity, phenolic compounds can also demonstrate some beneficial health functions such as antiviral, anti-inflammatory, anticarcinogenic, and antimicrobial activities [4-6]. Especially those found in essential oils derived 
from herbs and spices exhibit antimicrobial activity against Listeria monocytogenes, Salmonella typhimurium, Escherichia coli, and Staphylococcus aureus. Therefore these natural phytochemicals can be used to control microbial contamination of food products, delay their microbial spoilage, and eliminate undesirable pathogens. The antioxidant activity of herbs and spices containing polyphenols is based on their ability to neutralize free radicals, chelate transitional metals, and quench singlet and triplet oxygen by decomposition of peroxides [7]. For this reason, a good way to prolong stability and storage time of bakery products, in particular those containing fat, may be supplementation with natural antioxidants. Additionally to delaying the oxidation of lipids, these substances can protect other unsaturated compounds.

Many techniques are used to estimate the antioxidant effect of natural antioxidants on oxidative stability of fats. Among them are differential scanning calorimetry (DSC) [6], oxygen consumption test [8] or different accelerated aging tests $[9,10]$. As the oxidation of lipids proceeds through radical mechanism, electron paramagnetic resonance (EPR) spectroscopy can also be used to monitor the lipid oxidation [11-13]. This approach has been used in this study.

\section{Experimental}

\section{Materials}

Dried leaves of rosemary (Rosmarinus officinalis L.) and thyme (Thymus vulgaris L.) as well as ingredients for cookies: commercial soft wheat flour (type 550), sunflower oil, eggs, bakery shortening, baking powder, sugar, and salt were purchased from local food stores in Warsaw. PBN (N-tert-butyl-alpha-phenylnitrone) and BHA were purchased from Sigma-Aldrich Chemicals (Poznań, Poland). Ethanol and methanol were obtained from POCh (Gliwice, Poland), and chloroform from Chempur (Piekary Śląskie, Poland).

\section{Sample preparation}

Six sets of cookies were prepared. The thyme and rosemary extracts were prepared according to the method described by Kozłowska et al. [14]. The prepared extracts were added to the cookies at two different levels: $0.02 \%$ and $0.2 \%$ (relative to fat mixture - bakery shortening/sunflower oil, 1:1, w/w). Synthetic antioxidant BHA was added to the cookies at $0.02 \%$ level. Reference set of cookies was prepared without the addition of spice extracts or BHA. The cookies were prepared as described by Kozłowska et al. [15]. The lipid fractions from the cookies were extracted according to the Folch et al. procedure [16] immediately after baking and after 7 , 14 , and 21 days of storing the cookies at $60^{\circ} \mathrm{C}$. The extracted lipid samples were stored in refrigerated conditions until submitted to EPR spin trapping experiments.

\section{Spin trapping EPR}

For spin trapping experiments, the PBN spin trap has been used. The formation of free radicals during accelerated aging at elevated temperature was measured according to Velasco with modifications [12]. Briefly, $15 \mu \mathrm{l}$ of ethanolic solution of PBN (1.7 M) was added to $0.25 \mathrm{~g}$ of the lipid fraction of cookies. The solid samples were warmed to the temperature just over the melting point $\left(30-40^{\circ} \mathrm{C}\right)$ before sampling. The mixture was vortexed and put at $70^{\circ} \mathrm{C}$ into closed vessels. The EPR measurements were performed after 4, 5, 6, 7, and $24 \mathrm{~h}$ of accelerated aging. Each time, $50 \mu \mathrm{l}$ of the sample was taken. All EPR experiments were performed at room temperature. The spectra were recorded on a Magnettech MiniScope200 X-band spectrometer, with following settings: central field $333.5 \mathrm{mT}$, sweep $7.9 \mathrm{mT}$, modulation amplitude $0.1 \mathrm{mT}$, time constant $0.01 \mathrm{~s}$, sweep time $30 \mathrm{~s}$, three scans, and microwave power $5 \mathrm{~mW}$. All measurements were done in duplicate. The signal intensity was taken as the double integrate of the EPR spectrum. For integration, Origin 8 software was used. The integrated intensity of EPR signal after $6 \mathrm{~h}$ of accelerated aging as well as the ratio of EPR integrated intensities after 6 and $24 \mathrm{~h}$ $\left(I_{24 \mathrm{~h}} / I_{6 \mathrm{~h}}\right)$ of accelerated aging were taken as measures of oxidation susceptibility. Spectral parameters were obtained by simulations of EPR spectra, performed with EasySpin package for Matlab [17] using the Chili algorithm.

\section{Results}

\section{Immediately after baking}

For lipid fractions extracted from cookies just after baking, all the spectra were characteristic for PBN adducts of high molecular volume radicals in moderately viscous environment (Fig. 1). The EPR

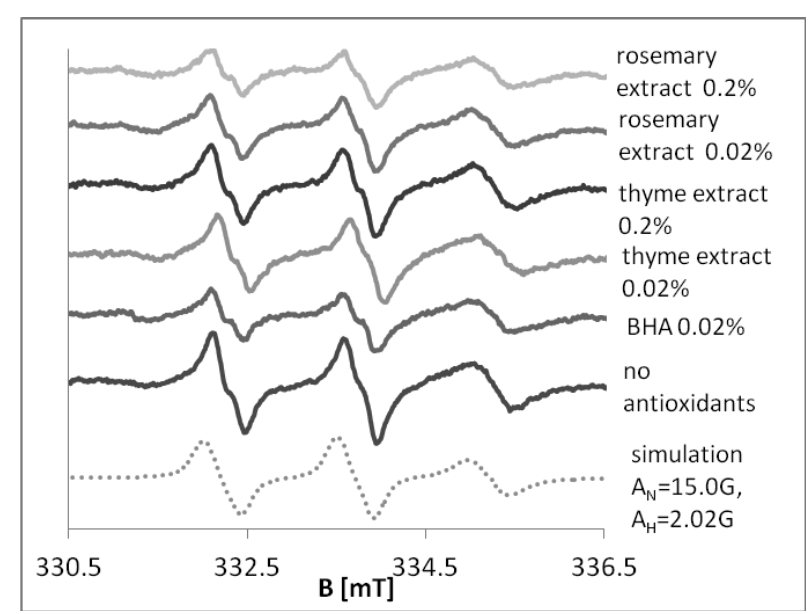

Fig. 1. Representative EPR spectra of PBN adducts registered after $6 \mathrm{~h}$ of accelerated aging at $70^{\circ} \mathrm{C}$ of lipid fractions extracted from cookies, and the exemplary simulated spectrum for the lipid fraction extracted from cookies with thyme extract in $0.02 \%$ concentration (dotted line). Lipid fractions of studied sets of cookies were extracted immediately after baking. 
lineshapes were similar to those of the spectra obtained for sunflower [13] and virgin olive [11] oils.

The obtained EPR parameters for lipid fractions extracted from cookies with added antioxidants $\left(A_{N}=15.0 \mathrm{G} ; A_{H}=1.8-2.1 \mathrm{G}, \tau=0.5-1.4 \mathrm{~ns}\right)$ were in agreement with those previously published for ultrasound-processed sunflower oil by Pingret et al. [13] $\left(A_{N}=15 \mathrm{G} ; A_{H}=1.85 \mathrm{G}\right)$, and by Jerzykiewicz et al. [19] for oleic acid $\left(A_{N}=15.0 \mathrm{G} ; A_{H}=1.9 \mathrm{G}\right)$. Such EPR parameters were ascribed to PBN-lipid radical adduct. In addition, the trend of the decreasing of the initial value of $A_{H}$ with time was observed, i.e., the values of $A_{H_{2} 4 \mathrm{~h}}$ were lower (in the range of $0.1 \mathrm{G}$ ) than the corresponding $A_{H_{6} \text {.h }}$. That could indicate the accumulation of adducts of longer chain fatty acids in the further stages of oxidation [18]. At the same time, the EPR parameters obtained for the control sample (no antioxidants) were $A_{N}=13.2 \mathrm{G}$ and $A_{H}=1.7 \mathrm{G}$ after $6 \mathrm{~h}$ and $A_{N}=13.5 \mathrm{G}$ and $A_{H}=1.7 \mathrm{G}$ after $24 \mathrm{~h}$ of accelerated aging of lipid fractions extracted from cookies at $70^{\circ} \mathrm{C}$. It could suggest that the presence of exogenous antioxidants influences the mechanism of lipid oxidation in the studied system. As a result, different radicals are formed in the presence of exogenous antioxidants, both synthetic (BHA), and natural (plant extracts) than in their absence.

\section{After 7 days of storage}

After 7 days of storage of cookies, the lineshapes of EPR spectra similar to these obtained for lipids extracted immediately after baking were observed only for lipid fractions extracted from cookies with the rosemary extract (both concentrations) (Fig. 2). The EPR lineshapes for all other lipid fractions can be ascribed to nitroxide radicals in the stronger restricted rotation regime (Fig. 2) ( $\tau$ in the range of 3-4 ns). Unfortunately, the determination of exact spectral parameters was difficult because of the large signal broadening. Still, for the control sample the obtained $A_{N}$ value $(13.5 \mathrm{G})$ was lower than these (around

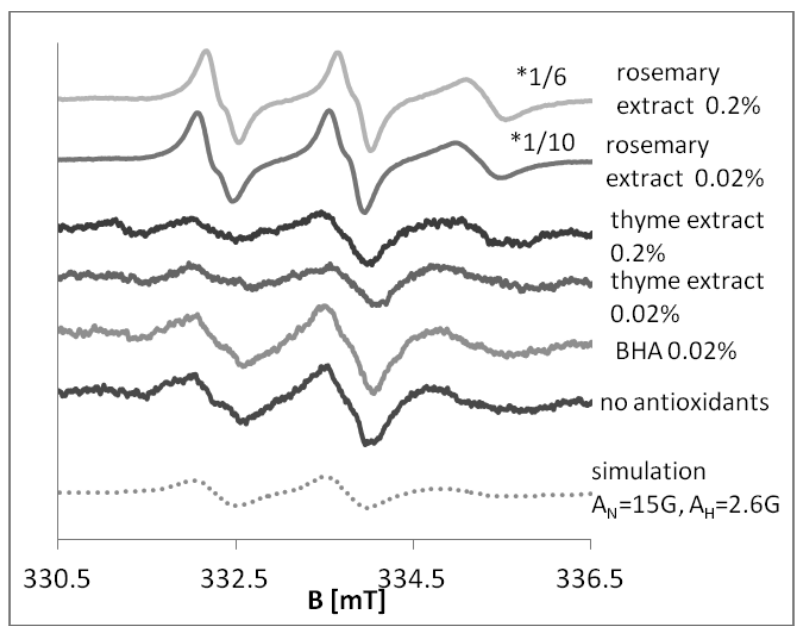

Fig. 2. Representative EPR spectra of PBN adducts registered after $6 \mathrm{~h}$ of accelerated aging at $70^{\circ} \mathrm{C}$ of lipid fractions extracted from cookies, and the exemplary simulated spectrum for the lipid fraction extracted from cookies with thyme extract in $0.02 \%$ concentration (dotted line). Lipid fractions of studied sets of cookies were extracted after 7 days of storage of cookies at $60^{\circ} \mathrm{C}$.
$15 \mathrm{G})$ for the samples with rosemary and thyme extracts. For the sample with BHA at this stage of storage of cookies the spectral parameters were close to these of the control sample (no antioxidants). This result can be tentatively ascribed to the significant degradation of BHA during storage of cookies in $60^{\circ} \mathrm{C}$, and therefore the domination of the same oxidation mechanism of extracted lipids during accelerated aging at $70^{\circ} \mathrm{C}$ in the presence of spin trap as for the lipid fraction of cookies without any antioxidants added (control sample).

\section{After 14 and 21 days of storage}

The EPR lineshape the same as for majority of samples after 7 days of storage was observed for all lipid fractions extracted from cookies after 14 and 21 days of storage of cookies.

This difference in spectral lineshapes of PBN adducts formed in lipid fractions extracted from cookies immediately after baking and after storage of cookies at $60^{\circ} \mathrm{C}$ can be ascribed to a difference in viscosity. All samples exhibiting the restricted rotation spectra were solid at room temperature, at which the EPR spectra were obtained; while all the lipid fractions extracted from cookies immediately after baking and lipid fractions extracted from cookies with the rosemary extract after 7 days of storage of cookies were fluid.

\section{Signal intensity}

During accelerated aging of lipid fractions of cookies in the presence of $\mathrm{PBN}$ at $70^{\circ} \mathrm{C}$, the increase in radical adducts concentration was approximately linear from 4 to 6 hours. However, for some samples the decrease of EPR signal intensity was observed after $7 \mathrm{~h}$ of the reaction of oil oxidation during accelerated aging at $70^{\circ} \mathrm{C}$. For this reason, the intensity after $6 \mathrm{~h}$ was taken as one of the indices of oxidative stability. Generally, the higher EPR intensity corresponds to the lower oxidative stability of the sample.

\section{Immediately after baking}

For the lipid fraction extracted from cookies immediately after baking, the lowest intensity of EPR spectra of radical adducts after $6 \mathrm{~h}$ of accelerated aging was observed for lipid fractions extracted from cookies with $0.2 \%$ of thyme and rosemary extracts, followed by BHA sample (Fig. 3a). For the lower concentration of thyme and rosemary extracts, the higher values of EPR signal intensities were observed. The control sample (no antioxidants) revealed the highest EPR signal intensity among all studies samples (Fig. 3a).

\section{After 7 days of storage}

After the storage of cookies for 7 days, the intensity of EPR spectra of radical adducts in lipid fractions after $6 \mathrm{~h}$ of accelerated aging at $70^{\circ} \mathrm{C}$ was lower for samples containing the thyme extract, in both 


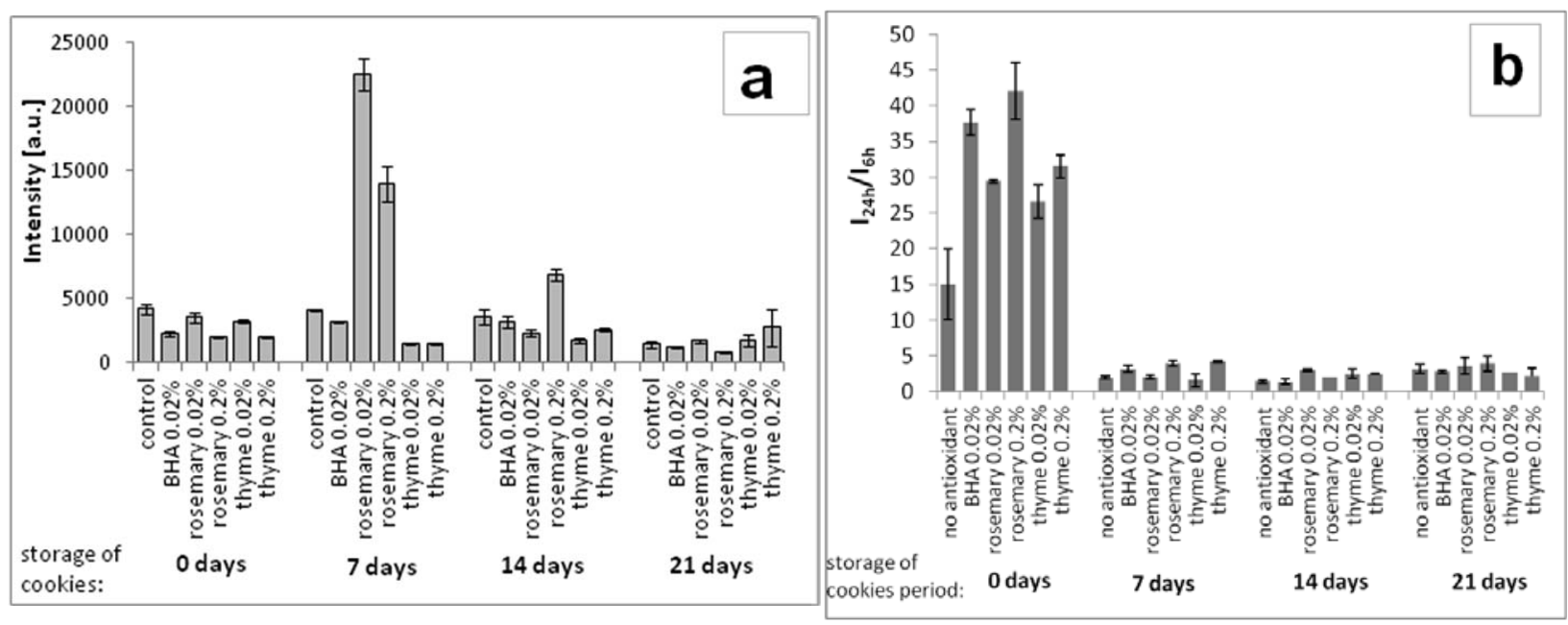

Fig. 3. The integrated intensity of EPR signal of lipid fractions extracted from cookies at different stages of storage at $60^{\circ} \mathrm{C}$ after $6 \mathrm{~h}$ of accelerated aging at $70^{\circ} \mathrm{C}$ (a) and the ratio of EPR integrated intensities after 6 and $24 \mathrm{~h}$ $\left(I_{24 \mathrm{~h}} / I_{6 \mathrm{~h}}\right)$ of accelerated aging $(\mathrm{b})$.

concentrations, and for a lipid fraction of cookies with BHA, than for the lipids extracted from cookies with no antioxidants added. On the other hand, lipids extracted from cookies with rosemary extract reveal drastic increase of EPR signal intensity as well as differences in spectral lineshape. This result together with the unchanged fluidity of the lipid fractions of cookies with rosemary extract during the first 7 days of storage of cookies could suggest different mechanism of reaction as compared to other samples, measured at the same time.

The lack of changes in fluidity during storage in samples with rosemary extract (measured immediately after baking and after 7 days of storage) can indicate that the rosemary extract was able to protect the unsaturated bonds in fatty acids in cookie matrix during first 7 days of storage at $60^{\circ} \mathrm{C}$. However, the increase of EPR signal intensity means the acceleration of oxidation reaction (Fig. 3a). Thus, it seems that the rosemary extract is the possible source of radical formation catalysts in extracted lipid fraction. It can be suggested that after antioxidants depletion, other substances present in rosemary extract take prooxidative action in elevated temperature, when the lipids are separated from the rest of cookie matrix.

\section{After 14 days of storage}

As it was mentioned earlier, after 14 days of storage, all samples had similar fluidity. As for the intensity of EPR signals of lipid fractions extracted from cookies after 14 days of storage of cookies, for lipid samples extracted from cookies with thyme extract (both $0.2 \%$ and $0.02 \%$ ) and the $0.02 \%$ concentration of rosemary extract it was lower than EPR intensity of the lipid fraction extracted from the control sample, i.e., cookies without antioxidants. For the BHA containing lipid sample the EPR intensity was approximately the same as for the lipids from the control sample. And for the lipid sample extracted from cookies with $0.2 \%$ concentration of rosemary extract the intensity was still much higher than for the control sample, though the difference was much less prominent than after 7 days of storage of cookies.

\section{After 21 days of storage}

The EPR signal intensity observed for lipid fractions extracted from cookies after 21 days of storage of cookies was the lowest in the whole experiment for all samples with exception of the thyme-enriched ones. It was probably due to the small amount of oxidation-prone substrates, such as unsaturated lipids, and the increased amount of free fatty acids. As shown by Jerzykiewicz et al. [19], the intensity of EPR signal of PBN lipid adducts was significantly lower for free fatty acids than for triglycerides and for saturated fatty acids than for unsaturated ones. In case of cookies with thyme extract, the intensity of obtained EPR spectra of lipid fraction of aged cookies was rather stable and did not depend noticeably on the time of storage of cookies, especially for the lower concentration of thyme extract. After 21 days of storage of cookies, the signal intensity for lipid fraction of cookies containing thyme extract was the highest among studied samples. The lipid fractions extracted from cookies with BHA and rosemary extract in $0.2 \%$ concentration gave the weakest signals, which were even lower than those given by lipid fraction extracted from cookies without antioxidant additives. It may suggest that the thyme extract exhibits antioxidant properties during prolonged storage of cookies, preserving the unsaturated fatty acids present which can form radicals in the spin trapping experiment. It confirms the results obtained from DSC measurements for the lipid fraction of cookies, as described by Kozlowska et al. [15].

\section{The ratio of EPR integrated intensities $\left(I_{24 h} / I_{6 h}\right)$}

It was previously shown that ratio $I_{24 \mathrm{~h}} / I_{6 \mathrm{~h}}$ could be used to describe the oxidative stability of oil samples [11]. It was found by Papadimitriou et al. [11] that $I_{24 h} / I_{6 \mathrm{~h}}$ is higher for virgin olive oil samples with 
higher oxidative stability. However, in our study the lipid fraction extracted from cookies with $0.2 \%$ concentration rosemary extract gave the highest result of $I_{24 \mathrm{~h}} / I_{6 \mathrm{~h}}$ value when extracted after 7 days of storage of cookies, equal within the margin of error to the result of lipids extracted from cookies with $0.2 \%$ thyme extract. It is contradictory to information given by the signal intensity as this sample, i.e. the lipid fraction extracted from cookies with $0.2 \%$ concentration rosemary extract gave very strong EPR signal which meant high instability. Therefore, it seems that the intensity ratio parameter is not a good choice for lipids extracted from cookies stored for prolonged time at elevated temperature.

\section{Conclusions}

According to the results obtained in this study, thyme extract can be used as a natural antioxidant source for the preparation of bakery products, as it inhibited the formation of radicals during lipid fraction oxidation comparably to BHA. However, based on spin-trapping experiment results, it seems that rosemary extract, though widely used as a lipid oxidation inhibitor, should be used with great care in fat-rich products exposed to high temperatures.

\section{References}

1. Grosso, N. R., \& Resurreccion, A. V. A. (2002). Predicting consumer acceptance ratings of cracker-coated and roasted peanuts from descriptive analysis and hexanal measurements. J. Food Sci., 67(4), 1530-1537. DOI: 10.1111/j.1365-2621.2002.tb10317.x.

2. Sasaki, Y. F., Kawaguchi, S., Kamaya, A., Ohshita, M., Kabasawa, K., Iwama, K., Taniguchi, K., \& Tsuda, S. (2002). The comet assay with 8 mouse organs: results with 39 currently used food additives. Mutat. Res. Genet. Toxicol. Environ. Mutagen., 519(1/2), 103-119. http://dx.doi.org/10.1016/S13835718(02)00128-6.

3. Labrador, V., Fernandez-Freire, P., Martin, J. M. P., \& Hazen, M. J. (2007). Cytotoxicity of butylated hydroxyanisole in Vero cells. Cell. Biol. Toxicol., 23, 189-199.

4. Boskou, D. (2006). Sources of natural antioxidants. Trends Food Sci. Technol., 17, 505-512.

5. Kuhlmann, A., \& Röhl, C. (2006). Phenolic antioxidant compounds produced by in vitro. Cultures of rosemary (Rosmarinus officinalis) and their antiinflammatory effect on lipopolysaccharide-activated microglia. Pharm. Biol., 44(6), 401-410. DOI: 10.1080/13880200600794063.

6. Shan, B., Cai, Y. -Z., Brooks, J. D., \& Corke, H. (2007). The in vitro antibacterial activity of dietary spice and medicinal herb extracts. Int. J. Food Microbiol., 117(1), 112-119. http://dx.doi.org/10.1016/j. ijfoodmicro.2007.03.003.

7. Chun, S. -S., Vattem, D. A., Lin, Y. -T., \& Shetty, K. (2005). Phenolic antioxidants from clonal oregano (Origanum vulgare) with antimicrobial activity against Helicobacter pylori. Process. Biochem., 40(2), 809-816. http://dx.doi.org/10.1016/j. procbio.2004.02.018.

8. Ulkowski, M., Musialik, M., \& Litwinienko, G. (2005). Use of differential scanning calorimetry to study lipid oxidation. 1. Oxidative stability of lecithin and linolenic acid. J. Agric. Food Chem., 53(23), 9073-9077. DOI: 10.1021/jf051289c.

9. Chen, Z. Y., Ratnayake, W. M. N., \& Cunnane, S. C. (1994). Oxidative stability of flaxseed lipids during baking. J. Am. Oil Chem. Soc., 71(6), 629-632. DOI: 10.1007/bf02540591.

10. Calligaris, S., Manzocco, L., Kravina, G., \& Nicoli, M. C. (2007). Shelf-life modeling of bakery products by using oxidation indices. J. Agric. Food Chem., 55(5), 2004-2009. DOI: 10.1021/jf063004h.

11. Papadimitriou, V., Sotiroudis, T. G., Xenakis, A. Sofikiti, N., Stavyiannoudaki, V., \& Chaniotakis, N. A. (2006). Oxidative stability and radical scavenging activity of extra virgin olive oils: An electron paramagnetic resonance spectroscopy study. Anal. Chim. Acta , 573/574, 453-458. http://dx.doi.org/10.1016/j. aca.2006.02.007.

12. Velasco, J., Andersen, M. L., \& Skibsted, L. H. (2004). Evaluation of oxidative stability of vegetable oils by monitoring the tendency to radical formation. A comparison of electron spin resonance spectroscopy with the Rancimat method and differential scanning calorimetry. Food Chem., 85(4), 623-632. http:// dx.doi.org/10.1016/j.foodchem.2003.07.020.

13. Pingret, D., Durand, G., Fabiano-Tixier, A. -S., Rockenbauer, A., Ginies, C., \& Chemat, F. (2012). Degradation of edible oil during food processing by ultrasound: Electron paramagnetic resonance, physicochemical, and sensory appreciation. J. Agric. Food Chem., 60(31), 7761-7768. DOI: 10.1021/jf301286f.

14. Kozłowska, M., Laudy, A. E., Starościak, B. J., Napiórkowski, A., Chomicz, L., \& Kazimierczuk, Z. (2010). Antimicrobial and antiprotozoal effect of sweet marjoram (Origanum majorana L.). Acta Sci. Pol., Hortorum Cultus, 9(4), 133-141.

15. Kozłowska, M., Żbikowska, A., Gruczyńska, E. Żontała, K., \& Półtorak, A. (2014). Effects of spice extracts on lipid fraction oxidative stability of cookies investigated by DSC. J. Therm. Anal. Calorim., 118(3), 1697-1705. DOI: $10.1007 /$ s10973-0144058-y.

16. Folch, J., Lees, M., \& Stanley, G. H. S. (1957). A simple method for the isolation and purification of total lipids from animal tissues. J. Biol. Chem., 226(1), 497-509.

17. Stoll, S., \& Schweiger, A. (2006). EasySpin, a comprehensive software package for spectral simulation and analysis in EPR. J. Magn. Reson., 178(1), 42-55. DOI: 10.1016/j.jmr.2005.08.013.

18. Janzen, E. G., \& Blackburn, B. J. (1968). Detection and identification of short-lived free radicals by an electron spin resonance trapping technique. J. Am. Chem. Soc., 90(21), 5909-5910. DOI: 10.1021/ ja01023a051.

19. Jerzykiewicz, M., Ćwielag-Piasecka, I., \& Jezierski, A (2013). Pro- and antioxidative effect of $\alpha$-tocopherol on edible oils, triglycerides and fatty acids. J. Am. Oil Chem. Soc., 90(6), 803-811. DOI: 10.1007/s11746013-2227-y. 